\title{
Performance of Cosmetic Ingredients Evaluated by Their Membrane Protection Efficiency
}

\author{
Daniela Rodrigues Silva*, Maidileyvis Castro Cabello*, Divinomar Severino, \\ Mauricio S. Baptista\# (1)
}

Departamento de Bioquímica, Instituto de Química, Universidade de São Paulo, São Paulo, Brazil

Email: "baptista@iq.usp.br

How to cite this paper: Silva, D.R., Cabello, M.C., Severino, D. and Baptista, M.S. (2021) Performance of Cosmetic Ingredients Evaluated by Their Membrane Protection Efficiency. Journal of Cosmetics, Dermatological Sciences and Applications, 11, 169-185.

https://doi.org/10.4236/jcdsa.2021.113016

Received: May 11, 2021

Accepted: July 13, 2021

Published: July 16, 2021

Copyright $\odot 2021$ by author(s) and Scientific Research Publishing Inc. This work is licensed under the Creative Commons Attribution International License (CC BY 4.0).

http://creativecommons.org/licenses/by/4.0/

\begin{abstract}
Plant extracts (PEs) are frequently used as ingredients in skin and hair care products to improve protection against the excess of sun exposure. PEs are usually selected based on their antioxidant capacity for reducing the damage in lipids and proteins, which are key molecules in the maintenance of skin homeostasis and hair integrity. Interestingly, biomolecules that do not have any antioxidant capacity can have a protective effect by physically adsorbing and inhibiting leakage of membranes. Our aims are: 1) to develop an experimental protocol that quantifies the level of membrane damage/protection; 2) to recommend an approach to compare the membrane protection efficiency of different PEs; 3) to briefly describe the mechanisms of membrane protection. Evaluation of membrane damage/protection is based on a phospholipid liposome system that signals the membrane rupture caused by photo-induced lipid oxidation. Carboxyfluorescein (CF) emission is monitored as a function of time (120 $\mathrm{min}$ ) in a controlled experimental setup, in which a certain level of membrane damage is induced by Dimethyl-Methylene Blue (DMMB) photosensitized oxidation during red-light irradiation. Percentage levels of membrane damage/protection are calculated by comparing the CF fluorescence level of DMMB-liposomes in the presence of varying concentrations of membrane-protective ingredients with that of surfactant-dissolved DMMB-liposomes. Membrane protectants reduce the level of CF fluorescence during irradiation of DMMB-liposomes. Gallic acid (GA) and trehalose (Tre) were used as standard compounds to compute the levels of protection by the antioxidant and physical adsorption mechanisms, respectively. The percentage of membrane protection was shown to be linearly proportional to the concentration of the standard protectants. The performance of PEs was measured and compared in terms of GA or Tre membrane protection equivalents. All extracted also had their antioxidant capacities evaluate by the anti-radical DPPH assay. The mem-
\end{abstract}

${ }^{\star}$ Contributed equally to the manuscript.

${ }^{\#}$ Corresponding author. 
brane protection efficiency parallels the anti-radical efficiency for extracts that have antioxidant activity. However, even extracts that are mainly enriched in polysaccharides and that do not have any measurable anti-radical capacity were able to protect membranes. We propose that these extracts protect membranes by the physical adsorption mechanism, in comparison with the protection observed by Tre and also based on the known effect of carbohydrates that can protect against membrane micro-fissures. The membrane damage assay described here can be used to evaluate and compare the performance of cosmetic ingredients with claims of maintaining lipid membrane integrity of skin and hair products.

\section{Keywords}

Photoprotection, Antioxidants, Sugars, Polyphenols, Flavonoids, Photochemistry

\section{Introduction}

The interaction of photons with absorbing molecules naturally present in human skin, here simply called photosensitizers (PS), can trigger a variety of photophysical and photochemical events [1]. For example, DNA itself absorbs Ultra-violet $B$ radiation (UVB, $280-320 \mathrm{~nm}$ ), triggering the formation of mutagenic photoadducts [2]. Among these processes, the formation of long-lifetime excited states (triplet excited states) facilitates several photosensitized oxidation reactions that can cause photoaging and other skin diseases [2] [3]. Uncontrolled lipid oxidation is also associated with many diseases, including cancer, Alzheimer and many inflammatory diseases [4]-[10].

The structure and function of human skin are highly dependent on the cutaneous barrier function, which avoids water loss as well as the entrance of external agents. Lipids exert a fundamental role in the barrier function by forming in the stratum corneum (SC) an orthorhombic, highly ordered, and densely packed, lamellar phase. The general lipid composition (by mass) of the SC is made of a mixture of ceramides $(\sim 50 \%)$, fatty acids $(10 \%-20 \%)$, and cholesterol $(\sim 25 \%)$ [11]. The ceramide portion is highly enriched in linoleic acid, a polyunsaturated fatty acid (PUFA) and consequently easily oxidized [12]. Indeed, lipid oxidation caused by sun exposure alters the lipid composition (formation of hydroperoxides and chain-truncated-aldehyde ceramides) of the SC [13] and considerable disturbances in the lipid packing and the barrier functions [14]. The basal layer and the dermis are tissues harboring living cells with bilayer lipid membranes, which provide physical barriers to separate the external from the internal environment of cells and organelles and mediate the entry and exit of diverse substances necessary to maintain cellular homeostasis. Lipid membranes are rich in conjugated double bonds and, therefore, are an easy target of photosensitized oxidation reactions [15]-[21]. Consequently, lipid integrity directly affects the 
homeostasis of superficial tissues [22].

Not only the skin but also the structure and function of hair depend on the composition and packing of lipids, which are made mainly of 18-methyleicosanoic acid (18-MEA) and free fatty acids, cholesterol, ceramides. Lipids exert a structural role in hair by self-organizing in phases called a cell membrane complex (CMC), which are present in both cuticle and cortical cells. CMC modulates the structure and the properties of both cuticle and cortical parts of hairs as well as the interface between them [23]. Unsaturated lipids present in the hair fibers are also prone to suffer oxidation by chemical treatments or by environmental insults, such as light exposure. Accumulation of lipid peroxides correlates with the loss of hair properties such as strength and shine [24].

The photoinduced lipid oxidation starts either by the Type I or the Type II mechanisms of photosensitized oxidation. In the Type I mechanism, electron or hydrogen abstraction from biological substrates form carbon-centered radicals, initiating a series of radical-chain reactions [25]. Most excited states will have enough energy to abstract hydrogens from unsaturated lipids carrying either a single double bond or several conjugated double bonds (PUFA). The photoinduced lipid oxidation mechanism differs from the radical-based auto-oxidation mechanism, which greatly depends on lipids carrying PUFA. After the first step of hydrogen abstraction, chains radical chain reactions can progress to the formation of peroxyl and alkoxyl radicals Alkoxyl radicals can suffer beta scission, leading to the accumulation of lipid truncated aldehydes [26] [27]. In the Type II mechanism, the excitation energy is transferred to molecular oxygen, resulting in the formation of singlet oxygen $\left({ }^{1} \mathrm{O}_{2}\right)$, which is highly electrophilic, adding to the double bond of biomolecules and in the case of lipids, forming hydroperoxides [26]. Both lipid truncated aldehydes and carboxylic acids as well as lipid hydroperoxides disturb significantly the packing order of lipids and the membranes' properties [16]. The lipid peroxidation reactions continue to propagate in the dark, with the beginning of a new chain of oxidations by peroxyl and alkoxyl radicals and the decomposition of lipid hydroperoxides into other intermediate radicals [28].

Antioxidant strategies are often used to prevent skin cancer and premature skin aging by inhibiting/spreading photo-oxidative reactions. Supplementation with compounds of plant origin and with pure molecules with recognized antioxidant potential is quite frequent [29]. Plant extracts effectively protect against the effects of sun exposure [30] [31] [32]. The action mechanism involves mainly the antioxidant effect, decreasing or inhibiting the damage generated by free radicals and/or other oxidizing compounds. Plant extracts' protective effect has motivated the cosmetic industry's interest, especially in understanding damage and protection mechanisms [30] [32]-[38]. In addition to the antioxidant effect, it has been shown recently that plant compounds can also have a protective effect by physically adsorption and mechanical protection against membrane rupture [39].

The level of membrane damage and membrane rupture can be evaluated by 
the Carboxyfluorescein (CF) assay in liposome suspension [40] [41]. Herein, we aim to report an experimental protocol that can evaluate membranes' damage by photosensitization and protection by different types of cosmetic ingredients. We also focus on assessing and quantifying the membrane protecting efficacy of several plant extracts (Figure 1) and on discussing the two most important mechanisms of membrane protection, i.e., antioxidant and physical adsorption.

\section{Materials and Methods}

\subsection{Reagents}

1,1'-diphenyl-2-picryl-hydrazyl (DPPH), 6-hydroxy-2,5,7,8 tetramethylchroman-2-carboxylic acid (Trolox), Carboxyfluorescein (CF) and Gallic Acid were purchased from Sigma-Aldrich. Tris-NaCl, Sephadex-G50, Chloroform, and 1,9-Dimethyl Methylene Blue (DMMB) were obtained from Merck. FolinCiocalteau was acquired from Haloquímica (São Paulo, Brazil). The vegetable extracts and soy lecithin were provided by Farma Service PE (São Paulo, Brazil).

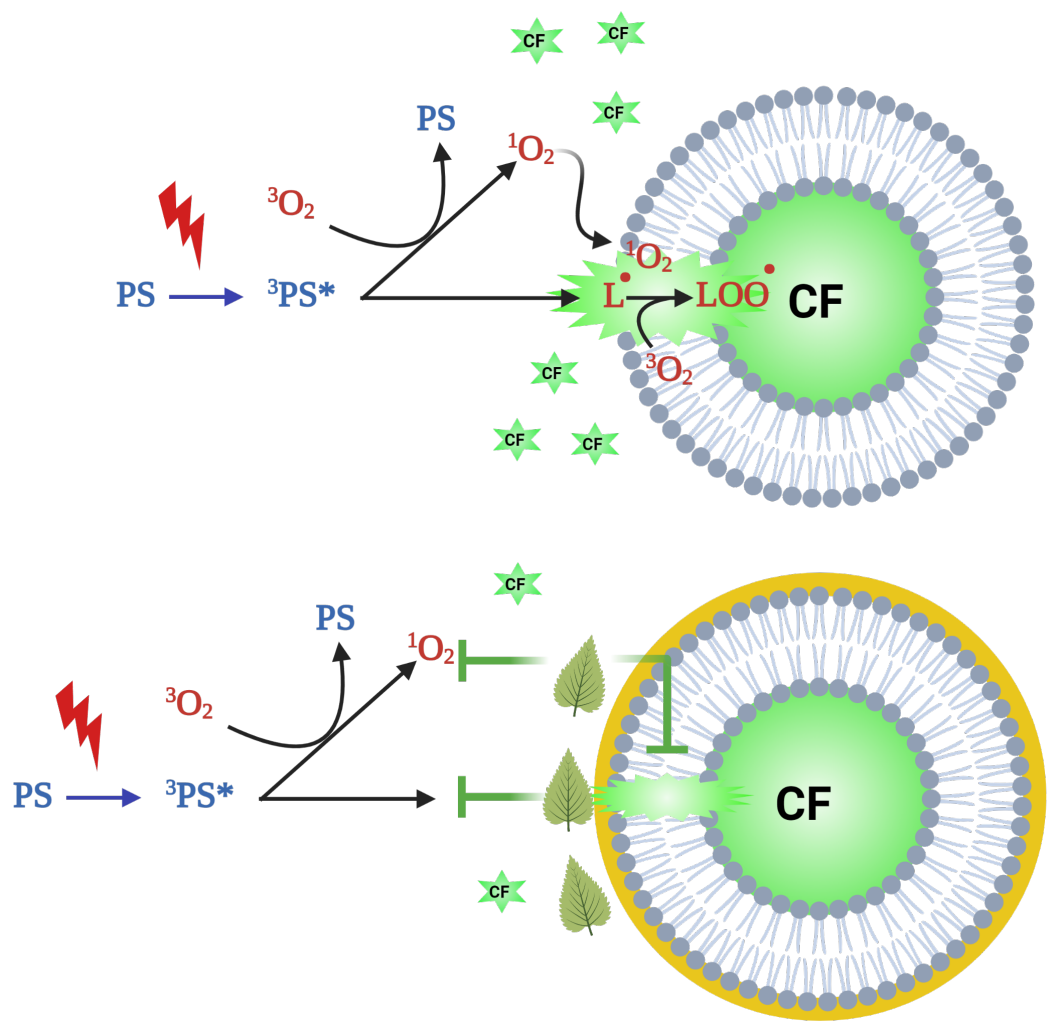

Figure 1. Proposed model for damage assessment and protection of membranes. Above: photo-induced reactions that cause lipid oxidation and membrane leakage. Below: the green leaves represent the anti-radical effect that avoids the generation reactive oxidant species and the propagation of the lipid peroxidation and the yellow shell represents carbohydrates that physically adsorb in the lipid head-groups and that avoid leakage. 


\subsection{Photosensitizer}

The 1,9-dimethyl methylene blue (DMMB) photosensitizer was solubilized in ethanol and diluted in water at around 30 times. The final concentration (15 $\mu \mathrm{M})$ was determined by absorption using a Shimadzu UV-VIS 2400 spectrophotometer.

\subsection{Preparation of the Carboxyfluorescein Solution}

The CF solution was prepared by adding $1.882 \mathrm{~g}$ of solid CF to $100 \mathrm{~mL}$ of an aqueous solution containing $60.57 \mathrm{~g}$ of Tris buffer. Sodium Hydroxide was added until the $\mathrm{pH}$ reached 8.0, and it was stored away from light.

\subsection{Preparation of Liposomes}

Multilamellar liposomes were prepared from soy lecithin with CF encapsulated in the internal compartment. Initially, Chloroform was added to soy lecithin in a test tube to obtain the final concentration of $30 \mathrm{mg} \cdot \mathrm{mL}^{-1}$. The solvent was removed with an Argon flow, while the tube was rotated in an inclined manner until obtaining a thin film on its walls. The film was hydrated with a CF solution and vortexed. After 10 minutes of sonication in an ultrasonic bath sonicator, the system was again vortexed. The suspension obtained was filtered on a Sephadex G-50 column equilibrated in $10 \mathrm{mM}$ Tris/ $\mathrm{HCl}(\mathrm{pH} \mathrm{8.0)}$ and $0.3 \mathrm{M} \mathrm{NaCl}$ to remove the free $\mathrm{CF}$ [42]. The fraction containing liposomes with $\mathrm{CF}$ encapsulated in the internal compartment was easily visualized in the Sephadex column and collected in a test tube.

\subsection{Quantification of Liposome Leakage}

The concentration of CF inside the liposomes is high enough to promote fluorescence self-quenching; therefore, an increase in CF fluorescence indicates leakage to the external solution [43] and can be used to measure membrane damage. The total fluorescence intensity of the CF released by lysis of the liposomes was measured with $5 \%(\mathrm{v} / \mathrm{v})$ Triton-X aqueous solution. The content of CF released from liposomes (percentage of release) was calculated by dividing the initial fluorescence intensity by the total intensity of the released CF.

$$
\% \text { Release }=\left[1-\left(\frac{\mathrm{FI}_{\text {initial }}}{\mathrm{FI}_{\text {total }}}\right)\right]
$$

The experiment to quantify the damage in the model membrane consisted of comparing the action of the photosensitizer (DMMB) in the permeabilization of the liposomes. This experiment was carried out in fluorescence plate reader of 96 wells clear bottom black, with 8 wells destined to the blank (only buffer), 8 wells destined to the control (only liposomes and buffer), and the rest to liposomes and variable amounts of the buffer, photosensitizer and vegetable compounds under study. The volume of liposome suspension added to the assay well chamber was always $7 \mu \mathrm{L}$, and the final volume was kept constant at $300 \mu \mathrm{L}$. The ir- 
radiation was carried out with LED's $\lambda_{\text {emission }}=639 \mathrm{~nm}$, in an arrangement determined to maximize light's dose and homogeneity. The fluorescence intensity was determined by using an Tecan Infinite M200 microplate reader (Männedorf, Switzerland), $\lambda_{\text {excitation }}=480 \mathrm{~nm} / \lambda_{\text {emission }}=517 \mathrm{~nm}$. One of the limitations of the assay proposed in this work is related to the possible presence of surfactants in the extracts. Amphiphilic compounds can directly interact and destroy the liposomes, thus preventing the measurements. Another factor that we could consider is the increase in the medium viscosity, since at higher viscosity the rate of substrate diffusion and membrane disruption is decreased [44]. As measurements are always made in comparison with controls, there will be no interference in the final results, as far as solution viscosity are the same for samples and controls.

\subsection{DPPH Radical Scavenging Capacity}

The radical scavenging capacity of vegetable extracts was evaluated by the suppression of the 2,2-Diphenyl-1-(2,4,6-trinitrophenyl)hydrazine radical (DPPH•), by using a classical procedure [45] with slight modifications. Briefly, aliquots with different concentrations of the sample extracts in ethanol were added to freshly prepared $0.1 \mathrm{mM}$ DPPH solutions. Each mixture was vortexed for a few seconds and incubated for 4 hours at room temperature. The absorbance was measure at $515 \mathrm{~nm}$ against a blank of ethanol using an Infinite M-200 Tecan microplate reader (Männedorf, Switzerland). Due to the action anti-oxidant capacity (usually donation of a hydrogen atom) of the PE, DPPH • is reduced, forming a yellow-colored diphenyl-picryl-hydrazine, with the consequent disappearance of absorption at $515 \mathrm{~nm}$. The absorption decay is monitored by the decrease in the solution absorbance at $515 \mathrm{~nm}$ [46]. 3 hours after the start the solution attain a steady state condition and the percentage of remaining DPPH $\bullet$ is calculated by using the formula:

$$
\% \mathrm{DPPH} \cdot \text { remaining }=\left(\frac{A_{f}}{A_{0}}\right) \times 100
$$

where $A_{0}$ and $A_{f}$ correspond to the absorbance at $515 \mathrm{~nm}$ of DPPH at initial and steady state respectively. $A_{f}$ value was obtained at the steady state region where absorbance did not depict further observable decreases.

The radical scavenging capacity was defined as the quantity of antioxidants needed to decrease the initial DPPH • concentration by $50 \%$ (efficient concentration $=\mathrm{EC}_{50}$ ), estimated by using a linear regression algorithm. All test analyzes were performed in triplicate. Trolox was used as a positive control and a calibration standard. A calibration curve was obtained in the concentration range of $0.0125-0.075 \mathrm{mg} \cdot \mathrm{mL}^{-1}$. After obtaining the $\mathrm{EC}_{50}$ of the solutions, was possible to obtain the amount of Trolox equivalent per kilogram of extract $\left(\mathrm{g} \cdot \mathrm{Kg}^{-1}\right)$ (TEAC), using the equation: TEAC $\left(\mathrm{EC}_{50}\right)=$ Trolox $(\mathrm{g}) /$ Extract $(\mathrm{Kg})$. Comparisons of anti-oxidant capacity were always performed after 4 hours of incubation time. 


\section{Results and Discussion}

\subsection{Efficiency and Mechanism of Membrane Protection}

In order to mimic the photosensitized oxidations occurring in lipid bilayers, we developed a liposome system carrying internalized self-quenched Carboxyfluorescein $(\mathrm{CF})$ and a red-light photosensitizer Dimethyl-methylene blue (DMMB), which can engage in both type I and type II photosensitized oxidation mechanisms [47] and, consequently, cause membrane damage by the formation of both lipid hydroperoxides and chain-truncated lipids [26] [27]. The suspension of Small Unilamellar Vesicles (SUV) with internalized and auto-suppressed CF and in the presence of the DMMB photosensitizer will be called DMMB-liposomes. A set of red-light LEDs irradiates DMMB-liposomes in order to trigger the photosensitized lipid oxidation. The kinetics of CF fluorescence is followed as a function of irradiation time and correlated with membrane leakage (Figure 2).

Light exposure prompts a sigmoid increase in the fluorescence intensity of the liposome suspension, due to the damage caused by the photo-induced lipid oxidation and the consequent membrane permeabilization (CF released in the bulk solution is no longer self-quenched) [27]. Note that the controls (buffer and liposome with buffer) exhibit irrelevant $\mathrm{CF}$ emission. The fluorescence level at $100 \%$ membrane leakage is obtained by dissolving the liposomes with Triton-X (see methods sections) and the CF emission intensity obtained after a 120-min irradiation periods is used to calculate the percentage of membrane damage at a certain experimental condition.

In order to quantify the efficiency of membrane protection, DMMB-liposomes were irradiated in the presence of a series of standard compounds and PEs. The PEs obtained from ethanolic solutions have a very rich and diverse chemical composition. Still, to evaluate their membrane protection efficiencies, we will consider two important groups: polyphenols and polysaccharides. Gallic Acid (GA), and Trehalose (Tre), respectively, were used as standard for polyphenols and a membrane protecting sugars [48]. Interestingly, there is a significant decrease in CF's emission with the increase in the concentrations of both GA and Tre, demonstrating membrane protection in both cases (Figure 3). It is essential to mention that the membrane protection measures carried out in this work are accurate since they are compared with control experiments. Therefore, external factors such as the presence of surfactants in the extract that directly affect the membrane can be easily detected.

As shown in Figure 4, the percentage of protection increases linearly with the GA and Tre concentrations. Note that there are strident differences in the concentration ranges. While GA protects the membrane in the micromolar concentration range, Tre works in the millimolar concentration range. This 3-order-ofmagnitude difference suggests that GA and Tre protect membranes by different mechanisms. 


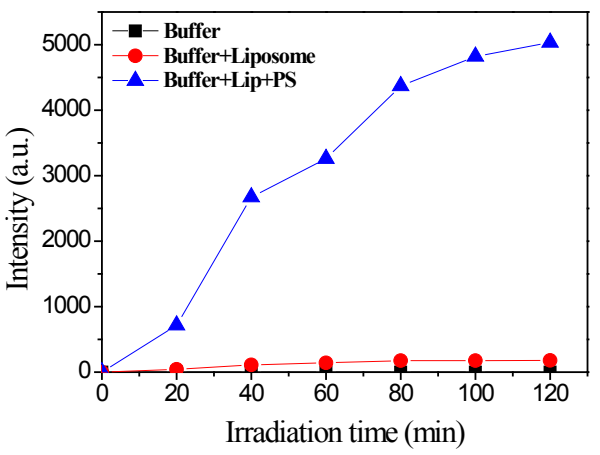

Figure 2. Fluorescence intensity as a function of irradiation time in lecithin liposomes with internalized CF (concentration) and $[\mathrm{DMMB}]=15 \mu \mathrm{M}, \mathrm{pH}=8.0 .\left(\lambda_{\mathrm{exc}}=480 \mathrm{~nm}, \lambda_{\mathrm{emis}}=517 \mathrm{~nm}\right)$.
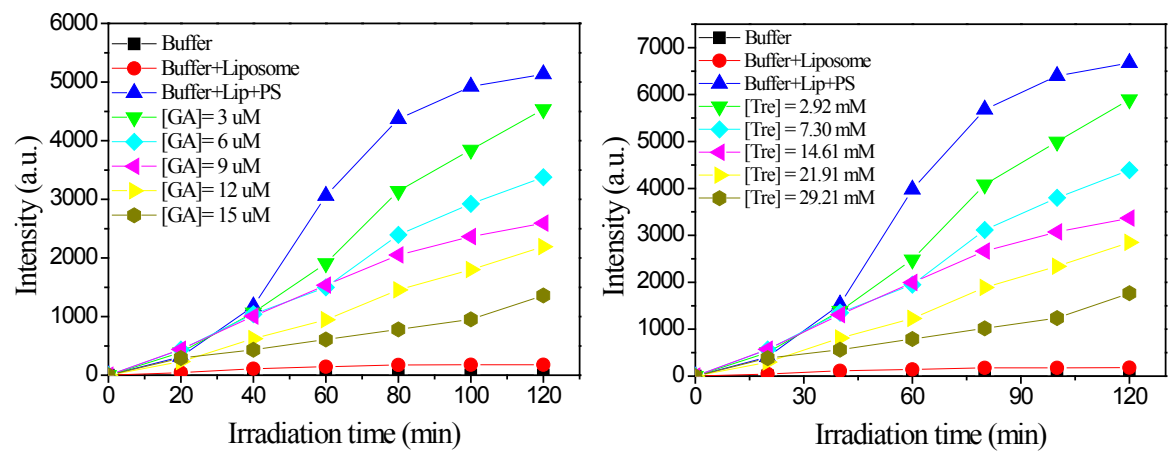

Figure 3. Carboxyfluorescein fluorescence intensity as a function of liposome irradiation time with varying concentrations of Gallic Acid (GA) and Trehalose (Tre).
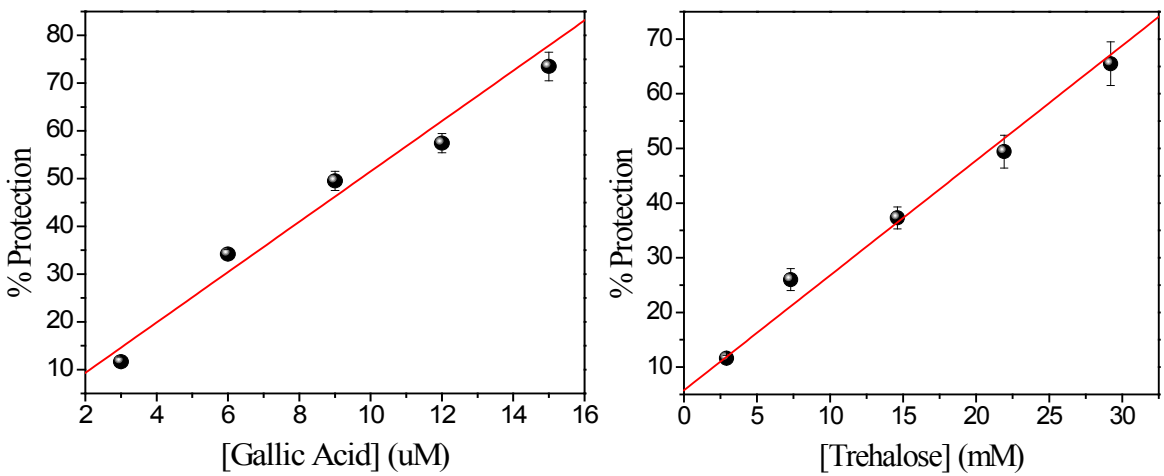

Figure 4. Percentage of membrane protection as a function of the concentration of standard vegetable extracts.

GA works by a typical anti-oxidant mechanism. By donating a hydrogen and forming a stable radical, and by avoiding the radical chain reaction's progression, GA avoids the lipid peroxidation and the membrane leakage [49] [50]. Contrarily, Tre does not have any known anti-oxidant activity, since it is a non-reducing disaccharide. Nevertheless, it also protects the membrane (Figure 4). Tre is known to protect the membrane structure by acting mainly by a physical adsorption mechanism. By efficiently interacting with the polar head groups of the lipids, Tre protects the membrane integrity by physically avoiding small 
changes in membrane conformation [51] [52]. This compound establishes hydrogen bonds with the carbonyl and phosphate groups and replaces water molecules from the lipid head group [53]. The interaction was computationally modeled, showing the high affinity and specificity of the lipid/trehalose interaction [54] [55]. The fact that both GA and Tre protect membranes (Figure 4) indicates that both mechanisms (anti-radical and physical adsorption) can be evaluated by this method.

\subsection{Vegetal Extracts as Membrane Protectants}

After standardizing the protocol to evaluate membrane damage/protection, attempts were made with different vegetable extracts to establish correlations between the properties of the extracts and their respective effectiveness in terms of protecting membranes.

The ability of all extracts to act as anti-radicals was evaluated by quantifying their capacity to suppress a relatively stable free radical, i.e.

2,2-Diphenyl-1-(2,4,6-trinitrophenyl)hydrazine $(\mathrm{DPPH} \bullet)$. $\mathrm{DPPH} \bullet$ is reproducibly annihilated by anti-oxidants, such as GA and Trolox, which can donate hydrogen atoms to DPPH $\bullet$. The radical scavenging activity of either pure compounds or vegetable extracts can be compared by evaluating the efficiency of radical suppression by a standard compound [56]. For this purpose, DPPH • UV-VIS absorption is followed as a function of time, in the absence and in the presence of antioxidant compounds and extracts. The anti-radical capacity of a vegetable extract is usually expressed as Trolox Equivalent Anti-radical (TEAC) per kilogram of extract $\left(\mathrm{g} \cdot \mathrm{Kg}^{-1}\right)$ (Table 1 ). Extracts that are known to carry anti-oxidant compounds present measurable TEAC values, varying from $\sim 0.6$ to $\sim 10$ grams Trolox equivalent per $\mathrm{Kg}$ of extract (Table 1, left column). Note also that the extracts that are basically made of polysaccharides either show minimal or unmeasurable TEAC values (Table 1, right column).

When adding these extracts to the DMMB-liposomes, it was observed that there are different levels of damage to the membrane during irradiation, depending on the extract and the concentration. The protective effect can be clearly noted, as the level of CF fluorescence decreased in the presence of several extracts. It is interesting to note that all vegetable extracts studied here have considerable membrane protection levels (Table 1).

In order to evaluate the significance of the free-radical scavenging mechanism, the percentage of membrane protection of each plant extract was plotted as a function of their respective TEAC value (Figure 5). Note that the efficiency of membrane protection increases linearly with the TEAC values. Linear regression with $\mathrm{R}^{2}=0.953$ was obtained by plotting membrane protection with TEAC values for the extracts F, C, G, W, P (Figure 5). Extracts with high anti-radical capacities, such as pomegranate and walnut, have a significantly higher capacity of membrane protection than those with low anti-radical activity (Fennel and Chamomile). 
Table 1. Percentage of membrane protection and TEAC values for different vegetable extracts.

\begin{tabular}{cccccc}
\hline Polyphenols & $\begin{array}{c}\% \\
\text { Protection }\end{array}$ & $\begin{array}{c}\text { TEAC } \\
\left(\mathrm{g} \cdot \mathrm{Kg}^{-1}\right)\end{array}$ & Saccharides & $\begin{array}{c}\% \\
\text { Protection }\end{array}$ & $\begin{array}{c}\text { TEAC } \\
\left(\mathrm{g} \cdot \mathrm{Kg}^{-1}\right)\end{array}$ \\
\hline Fennel 0.5\% & 60.47 & $0.58 \pm 0.08$ & Aloe Vera 0.5\% & 57.92 & without AOX \\
Chamomile 0.5\% & 64.90 & $1.12 \pm 0.10$ & Plantcol 0.5\% & 66.36 & without AOX \\
Guarana 0.1\% & 84.84 & $5.44 \pm 0.81$ & Tamariliz 0.5\% & 75.38 & $0.10 \pm 0.03$ \\
Walnut 0.1\% & 90.94 & $9.02 \pm 0.74$ & Alga Fucus 0.5\% & 46.92 & $0.17 \pm 0.04$ \\
Pomegranate 0.01\% & 94.33 & $9.87 \pm 0.93$ & & & \\
\hline
\end{tabular}

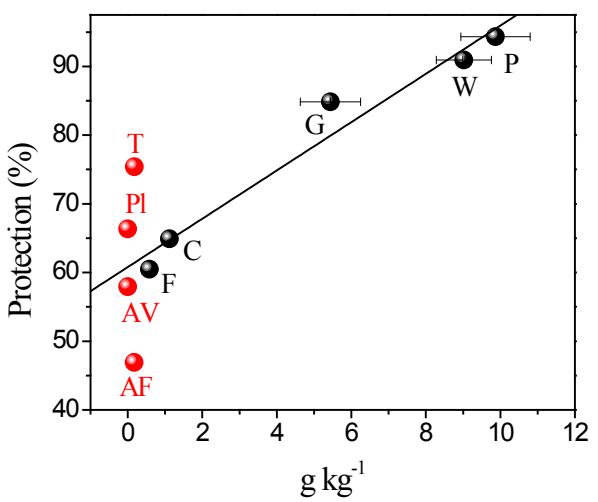

Figure 5. Percentage of membrane protection as a function of TEAC expressed in gram equivalent of trolox per kilogram of extracts. F: Fennel, C: Chamomile, G: Guarana, W: Walnut, P: Pomegranate, AF: Alga Fucus, AV: Aloe Vera, Pl: Plantcol, T: Tamariliz.

Several extracts have minimal or zero TEAC values but still offer membrane protection varying from $47 \%(\mathrm{AF})$ to $75 \%$ (T) (Table 1, write columns, data in red in Figure 5). All these extracts were enriched in naturally occurring polysaccharides. As discussed above, sugars generally have a low antiradical capacity since they protect the membranes by a different mechanism.

Finally, each plant extract's composition was determined as the equivalent of AG and Tre used as standards and correlated with the percentage of membrane protection (Table 2). This calculation considers the percentage of membrane protection, the equivalents of either GA and Tre, following the calibrations showed in Figure 4, and the concentrations used to attain the reached level of membrane protection. Note that Pomegranate extract achieved $94 \%$ of membrane protection at the very low concentration of $0.01 \%$, given a very high GA equivalent level, i.e., $32 \mathrm{~g} \cdot \mathrm{Kg}^{-1}$ extract. Note also that walnut and pomegranate have similar levels of anti-radical activities, as expressed by similar levels of Trolox equivalent (Table 1), but pomegranate has an at least 10-fold higher level of GA equivalent compared to walnut, indicating that other property, besides the anti-radical activity, is essential for the membrane protection. This other important property is likely to correlate with the level of membrane interaction of the active anti-radical ingredients [57]. 
Table 2. Membrane protection expressed as equivalents of Gallic Acid and Trehalose.

\begin{tabular}{cccccc}
\hline Polyphenols & $\begin{array}{c}\% \\
\text { Protection }\end{array}$ & $\begin{array}{c}\text { Equation GA } \\
\left(\mathrm{g} \cdot \mathrm{Kg}^{-1}\right)\end{array}$ & Saccharides & $\begin{array}{c}\% \\
\text { Protection }\end{array}$ & $\begin{array}{c}\text { Equation Tre } \\
\left(\mathrm{g} \cdot \mathrm{Kg}^{-1}\right)\end{array}$ \\
\hline Fennel 0.5\% & 60.47 & 0.40 & Aloe Vera 0.5\% & 57.92 & 1.74 \\
Chamomile 0.5\% & 64.90 & 0.44 & Plantcol 0.5\% & 66.36 & 2.04 \\
Guarana 0.1\% & 84.84 & 2.90 & Tamariliz 0.5\% & 75.38 & 2.36 \\
Walnut 0.1\% & 90.94 & 3.00 & Alga Fucus 0.5\% & 46.92 & 1.36 \\
Pomegranate 0.01\% & 94.33 & 32.0 & & & \\
\hline
\end{tabular}

It is also important to realize that even extracts with vanishing small TEAC levels protect membranes (physical adsorption mechanism). This activity can be quantified by the level of Tre equivalent. Note that Tamariliz extract was obtained from the Tamarindus fruits and has an exceptional level of membrane protection, and consequently, a high level of Tre equivalent $\left(\sim 2.4 \mathrm{~g} \cdot \mathrm{kg}^{-1}\right)$. This high level is likely to be a consequence of the extraction process that favors the presence of high molecular weight polysaccharides in its composition.

As mentioned above, lipid photooxidation occurs by both a Type I and Type II mechanism. The direct reactions between the excited triplet state of the photosensitizer $\left({ }^{3} \mathrm{PS}^{\star}\right)$ and the substrate (in this case, the lipid double bond) are usually electron transfers, forming radicals that initiate a series of chain reactions. On the other hand, the excited state generates a mediator species $\left({ }^{1} \mathrm{O}_{2}\right)$ which diffuses and reacts with unsaturated lipids. Oxidation reactions significantly alter the structure and properties of the membrane. The accumulation of lipid truncate aldehydes allows the formation of nanopores [27], causing the release of the internal fluorophore (CF) in the liposome to the external environment (Figure 6(A)). Extracts containing polyphenols neutralize free radicals and suppress excited states due to their antioxidant capacity, interrupting lipid peroxidation and avoiding membrane leakage [58] (Figure 6(B)). Indeed, antioxidants have been increasingly used in formulations of sun care products by protecting skin cells from the redox imbalance [59]. On the other hand, those enriched in polysaccharides bind to the membrane through a physical adsorption mechanism; that is, they interact directly with the phospholipids' hydrophilic part [48], preserving the bilayer structure even when oxidative damage occurs at the hydrophobic tails (Figure $6(\mathrm{C})$ ). It is worth mentioning the great potential of Tre, which stimulates autophagy and exhibits micro-fissures on membranes during freezing and reheating [60] [61]. As shown here, Tre also efficiently protects membranes against photo-induced membrane damage, even though it has no anti-radical effect. Another interesting example is that Aloe Vera (Aloe barbadensis) extracts protect keratinocytes against cell death induced by solar irradiation [62]. Aloe Vera exhibited the remarkable ability to reduce photodamage in vitro and in vivo, although it has no anti-radical properties. The protection afforded by Aloe Vera was associated with maintaining the integrity of the membrane of lysosomes [62]. 


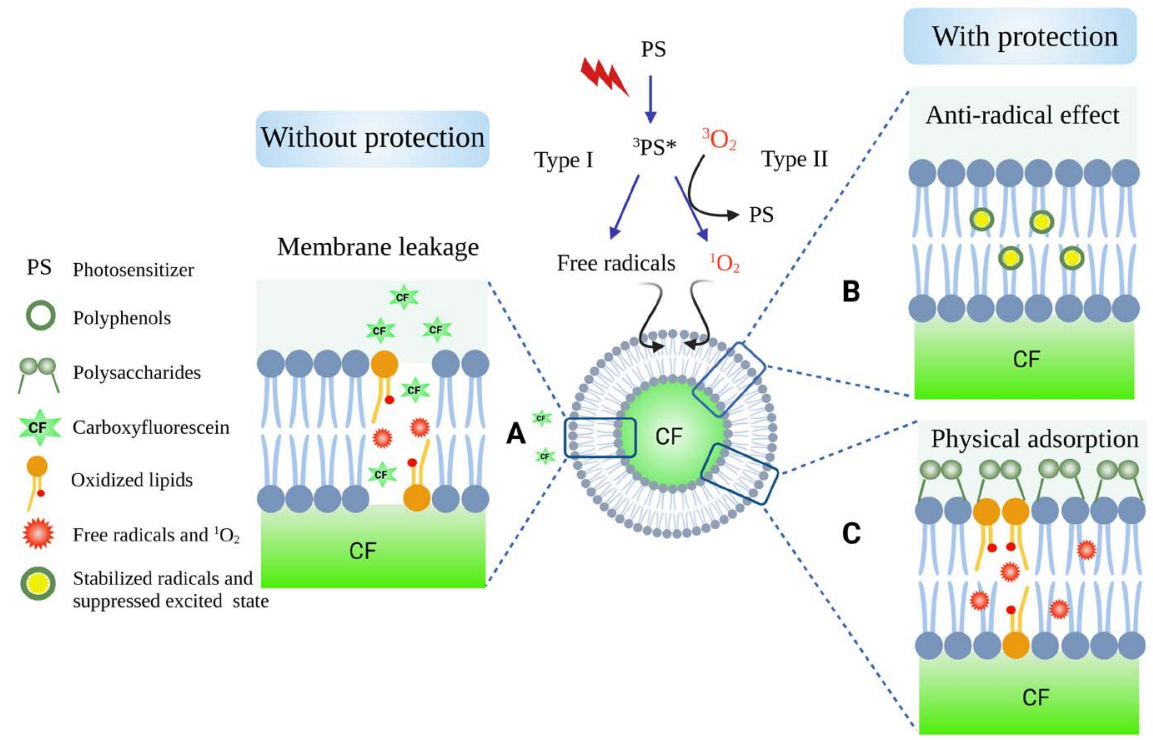

Figure 6. Schematic representation of the photoinduced lipid oxidation (A) and membrane protection by the mechanisms of anti-radical (B) and physical adsorption (C) mechanisms.

Although the phytotherapic compounds are commonly used as photo-protectors against the harmful effects of overexposure to sun light, the mechanisms involved are not precisely known. Therefore, we anticipate that the estimation of the performance of the PEs in terms of membrane protection efficiency will help to find solutions that maximize performance with regard to the protection of the lipid components of the skin and hair.

\section{Conclusion}

The quantification of the membrane protection efficiency provides a novel method to quantify ingredients aimed for sun care and antiaging strategies. Membrane protection efficiency was quantified initially as a function of the concentration of standard compounds, based on the anti-radical effect (Equation GA and TEAC) and on the physical adsorption on the membrane head groups (Tre). The PEs enriched either in polyphenols or in polysaccharides were analyzed and compared in terms of their efficiency to membrane protection. All examined extracts protect the membrane from photoinduced damage, indicating that both the anti-radical and the physical adsorption mechanisms can protect the integrity of membranes. The GA or Tre equivalents associated to vegetable extracts' capacity to providing membrane protection provide appropriate ways of quantifying and comparing PEs. The membrane protection assay is a simple and low-cost method, which can be used in the cosmetic industry to sustain new claims such as the protection of SC and of CMC lipids, respectively, in skin and hair.

\section{Acknowledgements}

The authors would like to thank funding by FarmaService Bioextract, Capes, 


\section{CNPq, FAPESP (CEPID-REDOXOMA 2013/07937-8).}

\section{Conflicts of Interest}

The authors declare no conflicts of interest regarding the publication of this paper.

\section{References}

[1] Ahmad, S.I. (2017) Ultraviolet Light, Chromophores, Reactive Oxygen Species, and Human Health. In: Reactive Oxygen Species in Biology and Human Health, CRC Press, Boca Raton, 567. https://doi.org/10.1201/b20228

[2] de Assis, L.V.M., Tonolli, P.N., Moraes, M.N., Baptista, M.S. and de Lauro Castrucci, A.M. (2021) How Does the Skin Sense Sun Light? An Integrative View of Light Sensing Molecules. Journal of Photochemistry and Photobiology C: Photochemistry Reviews, 47, Article ID: 100403.

https://doi.org/10.1016/j.jphotochemrev.2021.100403

[3] Bolton, P.D., Hall, F.M. and Reece, I.H. (1967) Effects of Substituents on the Thermodynamic Functions of Ionisation of Meta-Substituted Phenols. Journal of the Chemical Society B, 709-712. https://doi.org/10.1039/j29670000709

[4] Bhatia, V. and Sharma, S. (2021) Role of Mitochondrial Dysfunction, Oxidative Stress and Autophagy in Progression of Alzheimer's Disease. Journal of the Neurological Sciences, 421, Article ID: 117253. https://doi.org/10.1016/j.jns.2020.117253

[5] Fracassi, A., et al. (2021) Oxidative Damage and Antioxidant Response in Frontal Cortex of Demented and Nondemented Individuals with Alzheimer's Neuropathology. Journal of Neuroscience, 41, 538-554. https://doi.org/10.1523/JNEUROSCI.0295-20.2020

[6] Pisoschi, A.M., et al. (2021) Oxidative Stress Mitigation by Antioxidants-An Overview on Their Chemistry and Influences on Health Status. European Journal of Medicinal Chemistry, 209, Article ID: 112891.

https://doi.org/10.1016/j.ejmech.2020.112891

[7] Guarneri, F., Custurone, P., Papaianni, V. and Gangemi, S. (2021) Involvement of RAGE and Oxidative Stress in Inflammatory and Infectious Skin Diseases. Antioxidants, 10, 82. https://doi.org/10.3390/antiox10010082

[8] Ikwegbue, P.C., Masamba, P., Oyinloye, B.E. and Kappo, A.P. (2018) Roles of Heat Shock Proteins in Apoptosis, Oxidative Stress, Human Inflammatory Diseases, and Cancer. Pharmaceuticals, 11, 2. https://doi.org/10.3390/ph11010002

[9] Reuter, S., Gupta, S.C., Chaturvedi, M.M. and Aggarwal, B.B. (2010) Oxidative Stress, Inflammation, and Cancer: How Are They Linked? Free Radical Biology and Medicine, 49, 1603-1616. https://doi.org/10.1016/j.freeradbiomed.2010.09.006

[10] Gaschler, M.M. and Stockwell, B.R. (2017) Lipid Peroxidation in Cell Death. Biochemical and Biophysical Research Communications, 482, 419-425. https://doi.org/10.1016/j.bbrc.2016.10.086

[11] van Smeden, J., Janssens, M., Gooris, G.S. and Bouwstra, J.A. (2014) The Important Role of Stratum Corneum Lipids for the Cutaneous Barrier Function. Biochimica et Biophysica Acta (B BA)-Molecular and Cell Biology of Lipids, 1841, 295-313. https://doi.org/10.1016/j.bbalip.2013.11.006

[12] López-Duarte, A.L. and Vidal-Quintanar, R.L. (2009) Oxidation of Linoleic Acid as a Marker for Shelf Life of Corn Flour. Food Chemistry, 114, 478-483.

https://doi.org/10.1016/j.foodchem.2008.09.105 
[13] Gruber, F., Kremslehner, C. and Narzt, M.S. (2019) The Impact of Recent Advances in Lipidomics and Redox Lipidomics on Dermatological Research. Free Radical Biology and Medicine, 144, 256-265. https://doi.org/10.1016/j.freeradbiomed.2019.04.019

[14] Biniek, K., Levi, K. and Dauskardt, R.H. (2012) Solar UV Radiation Reduces the Barrier Function of Human Skin. Proceedings of the National Academy of Sciences of the United States of America, 109, 17111-17116. https://doi.org/10.1073/pnas.1206851109

[15] Roqueiro, G., et al. (2010) Effects of Photooxidation on Membrane Integrity in Salix nigra Seeds. Annals of Botany, 105, 1027-1034. https://doi.org/10.1093/aob/mcq067

[16] Tsubone, T.M., Baptista, M.S. and Itri, R. (2019) Understanding Membrane Remodelling Initiated by Photosensitized Lipid Oxidation. Biophysical Chemistry, 254, Article ID: 106263. https://doi.org/10.1016/j.bpc.2019.106263

[17] Marszałek, A. and Puszczało, E. (2020) Effect of Photooxidation on Nanofiltration Membrane Fouling during Wastewater Treatment from the Confectionery Industry. Water (Switzerland), 12, 793. https://doi.org/10.3390/w12030793

[18] Itri, R., Junqueira, H.C., Mertins, O. and Baptista, M.S. (2014) Membrane Changes under Oxidative Stress: The Impact of Oxidized Lipids. Biophysical Reviews, 6, 47-61. https://doi.org/10.1007/s12551-013-0128-9

[19] Tsubone, T.M., et al. (2017) Enhanced Efficiency of Cell Death by Lysosome-Specific Photodamage. Scientific Reports, 7, Article No. 6734.

https://doi.org/10.1038/s41598-017-06788-7

[20] Almeida, A.M., Oliveira, O.N. and Aoki, P.H.B. (2019) Role of Toluidine Blue-O Binding Mechanism for Photooxidation in Bioinspired Bacterial Membranes. Langmuir, 35, 16745-16751. https://doi.org/10.1021/acs.langmuir.9b03045

[21] Ma, J., et al. (2017) Comparative Analysis of Ultrastructure, Antioxidant Enzyme Activities, and Photosynthetic Performance in Rice Mutant 812HS Prone to Photooxidation. Photosynthetica, 55, 568-578.

https://doi.org/10.1007/s11099-016-0669-9

[22] Casares, D., Escribá, P.V. and Rosselló, C.A. (2019) Membrane Lipid Composition: Effect on Membrane and Organelle Structure, Function and Compartmentalization and Therapeutic Avenues. International Journal of Molecular Sciences, 20, 2167. https://doi.org/10.3390/ijms20092167

[23] McMullen, R.L., et al. (2013) Determination of Physicochemical Properties of Delipidized Hair. Journal of Cosmetic Science, 64, 355-370.

[24] Marsh, J.M., et al. (2018) Role of Internal Lipids in Hair Health. Journal of Cosmetic Science, 69, 347-356.

[25] Baptista, M.S., et al. (2017) Type I and Type II Photosensitized Oxidation Reactions: Guidelines and Mechanistic Pathways. Photochemistry and Photobiology, 93, 912-919. https://doi.org/10.1111/php.12716

[26] Bacellar, I.O.L., et al. (2014) Membrane Damage Efficiency of Phenothiazinium Photosensitizers. Photochemistry and Photobiology, 90, 801-813.

https://doi.org/10.1111/php.12264

[27] Bacellar, I.O.L., Oliyeira, M.C., Dantas, L.S., Costa, E.B., Junqueira, H.C., Martins, W.K., Durantini, A.M., Cosa, G., Di Mascio, P., Wainwright, M., Miotto, R., Cordeiro, R.M., Miyamoto, S. and Baptista, M.S. (2018) Photosensitized Membrane Permeabilization Requires Contact-Dependent Reactions between Photosensitizer and Lipids. Journal of the American Chemical Society, 140, 9606-9615.

https://doi.org/10.1021/jacs.8b05014 
[28] Yalcinkaya, T., Uzilday, B., Ozgur, R., Turkan, I. and Mano, J. (2019) Lipid Peroxidation-Derived Reactive Carbonyl Species (RCS): Their Interaction with ROS and Cellular Redox during Environmental Stresses. Environmental and Experimental Botany, 165, 139-149. https://doi.org/10.1016/j.envexpbot.2019.06.004

[29] Rogowski-Tylman, M., Narbutt, J., Woźniacka, A. and Lesiak, A. (2016) Molecular Aspects of Skin Aging. Literature Review. Przeglad Dermatologiczny, 103, 139-142. https://doi.org/10.5114/dr.2016.59136

[30] Twilley, D., et al. (2021) Ethanolic Extracts of South African Plants, Buddleja saligna Willd. and Helichrysum odoratissimum (L.) Sweet, as Multifunctional Ingredients in Sunscreen Formulations. South African Journal of Botany, 137, 171-182. https://doi.org/10.1016/j.sajb.2020.10.010

[31] Cefali, L.C., Ataide, J.A., Moriel, P., Foglio, M.A. and Mazzola, P.G. (2016) Plant-Based Active Photoprotectants for Sunscreens. International Journal of Cosmetic Science, 38, 346-353. https://doi.org/10.1111/ics.12316

[32] Sutar, M.P. and Chaudhari, S.R. (2020) Screening of in Vitro Sun Protection Factor of Some Medicinal Plant Extracts by Ultraviolet Spectroscopy Method. Journal of Applied Biology \& Biotechnology, 8, 48-53.

[33] Kalouta, K., Eleni, P., Boukouvalas, C., Vassilatou, K. and Krokida, M. (2020) Dynamic Mechanical Analysis of Novel Cosmeceutical Facial Creams Containing Nano-Encapsulated Natural Plant and Fruit Extracts. Journal of Cosmetic Dermatolo$g y, 19,1146-1154$. https://doi.org/10.1111/jocd.13133

[34] Naser, W. (2021) The Cosmetic Effects of Various Natural Biofunctional Ingredients against Skin Aging: A Review. International Journal of Applied Pharmaceutics, 13, 10-18. https://doi.org/10.22159/ijap.2021v13i1.39806

[35] Benoit, C., Virginie, C. and Boris, V. (2021) The Use of NADES to Support Innovation in the Cosmetic Industry. In: Advances in Botanical Research, Elsevier, Amsterdam, Vol. 97, 309-332. https://doi.org/10.1016/bs.abr.2020.09.009

[36] Malinowska, P. (2014) Effect of Flavonoids Content on Antioxidant Activity of Commercial Cosmetic Plant Extracts. Herba Polonica, 59, 63-75.

https://doi.org/10.2478/hepo-2013-0017

[37] Desam, N.R. and Al-Rajab, A.J. (2021) The Importance of Natural Products in Cosmetics. In: Pal, D. and Nayak, A.K., Eds., Bioactive Natural Products for Pharmaceutical Applications, Advanced Structured Materials, Vol. 140, Springer, Berlin, 643-685. https://doi.org/10.1007/978-3-030-54027-2_19

[38] Morganti, P. (2016) Green Ingredients in Cosmetic Dermatology. Molecular Aspects of Ingredients and Carriers. Journal of Applied Cosmetology, 34, 65-79.

[39] St-Pierre, A., et al. (2019) Study of Antioxidant Properties of Thylakoids and Application in UV Protection and Repair of UV-Induced Damage. Journal of Cosmetic Dermatology, 18, 1980-1991. https://doi.org/10.1111/jocd.12936

[40] Gregoriadis, G., et al. (2019) Self-Quenching of Carboxyfluorescein Fluorescence: Uses in Studying Liposome Stability and Liposome-Cell Interaction. In: Liposome Technology, CRC Press, Boca Raton, 183-204.

[41] Zhang, D., Okada, S. and Yasuda, T. (1993) A Carboxyfluorescein-Enveloping Liposome as a Physicochemical Damage Model of the Biomembrane for the Study of Lipid Peroxidation. Journal of Clinical Biochemistry and Nutrition, 14, 83-90. https://doi.org/10.3164/jcbn.14.83

[42] Martins, R.M., et al. (2008) A Short Proregion of Trialysin, a Pore-Forming Protein of Triatoma infestans Salivary Glands, Controls Activity by Folding the N-Terminal Lytic Motif. The FEBS Journal, 275, 994-1002. 
https://doi.org/10.1111/j.1742-4658.2008.06260.x

[43] Weinstein, J.N., Yoshikami, S., Henkart, P., Blumenthal, R. and Hagins, W.A. (1977) Liposome-Cell Interaction: Transfer and Intracellular Release of a Trapped Fluorescent Marker. Science, 195, 489-492. https://doi.org/10.1126/science.835007

[44] Karal, M.A.S., Islam, M.K. and Mahbub, Z.B. (2020) Study of Molecular Transport through a Single Nanopore in the Membrane of a Giant Unilamellar Vesicle Using COMSOL Simulation. European Biophysics Journal, 49, 59-69. https://doi.org/10.1007/s00249-019-01412-0

[45] Blois, M.S. (1958) Antioxidant Determinations by the Use of a Stable Free Radical. Nature, 181, 1199-1200. https://doi.org/10.1038/1811199a0

[46] Liang, N. and Kitts, D.D. (2014) Antioxidant Property of Coffee Components: Assessment of Methods that Define Mechanisms of Action. Molecules, 19, 19180-19208. https://doi.org/10.3390/molecules191119180

[47] Martins, W.K., et al. (2019) Parallel Damage in Mitochondria and Lysosomes Is an Efficient Way to Photo-Induce Cell Death. Autophagy, 15, 259-279.

https://doi.org/10.1080/15548627.2018.1515609

[48] Leslie, S.B., Israeli, E., Lighthart, B., Crowe, J.H. and Crowe, L.M. (1995) Trehalose and Sucrose Protect Both Membranes and Proteins in Intact Bacteria during Drying. Applied and Environmental Microbiology, 61, 3592-3597.

https://doi.org/10.1128/aem.61.10.3592-3597.1995

[49] Badhani, B., Sharma, N. and Kakkar, R. (2015) Gallic Acid: A Versatile Antioxidant with Promising Therapeutic and Industrial Applications. RSC Advances, 5, 27540-27557. https://doi.org/10.1039/C5RA01911G

[50] Zhao, M.T., et al. (2021) Gallic Acid and Its Alkyl Esters Emerge as Effective Antioxidants against Lipid Oxidation during Hot Air Drying Process of Ostrea talienwhanensis. LWT, 139, Article ID: 110551. https://doi.org/10.1016/j.lwt.2020.110551

[51] Tapia, H. and Koshland, D.E. (2014) Trehalose Is a Versatile and Long-Lived Chaperone for Desiccation Tolerance. Current Biology, 24, 2758-2766. https://doi.org/10.1016/j.cub.2014.10.005

[52] Eleutherio, E., Panek, A., De Mesquita, J.F., Trevisol, E. and Magalhães, R. (2015) Revisiting Yeast Trehalose Metabolism. Current Genetics, 61, 263-274. https://doi.org/10.1007/s00294-014-0450-1

[53] Elbein, A.D., Pan, Y.T., Pastuszak, I. and Carroll, D. (2003) New Insights on Trehalose: A Multifunctional Molecule. Glycobiology, 13, 17-27. https://doi.org/10.1093/glycob/cwg047

[54] Pereira, C.S., Lins, R.D., Chandrasekhar, I., Freitas, L.C.G. and Hünenberger, P.H. (2004) Interaction of the Disaccharide Trehalose with a Phospholipid Bilayer: A Molecular Dynamics Study. Biophysical Journal, 86, 2273-2285.

https://doi.org/10.1016/S0006-3495(04)74285-X

[55] Pereira, C.S. and Hünenberger, P.H. (2006) Interaction of the Sugars Trehalose, Maltose and Glucose with a Phospholipid Bilayer: A Comparative Molecular Dynamics Study. The Journal of Physical Chemistry B, 110, 15572-15581.

https://doi.org/10.1021/jp0607891

[56] Van Den Berg, R., Haenen, G.R.M.M., Van Den Berg, H. and Bast, A. (1999) Applicability of an Improved Trolox Equivalent Antioxidant Capacity (TEAC) Assay for Evaluation of Antioxidant Capacity Measurements of Mixtures. Food Chemistry, 66, 511-517. https://doi.org/10.1016/S0308-8146(99)00089-8

[57] Niki, E. and Noguchi, N. (2004) Dynamics of Antioxidant Action of Vitamin E. 
Accounts of Chemical Research, 37, 45-51. https://doi.org/10.1021/ar030069m

[58] Lúcio, M., Ferreira, H., Lima, J.L.F.C. and Reis, S. (2007) Use of Liposomes to Evaluate the Role of Membrane Interactions on Antioxidant Activity. Analytica Chimica Acta, 597, 163-170. https://doi.org/10.1016/j.aca.2007.06.039

[59] Freitas, J.V., Junqueira, H.C., Martins, W.K., Baptista, M.S. and Gaspar, L.R. (2019) Antioxidant Role on the Protection of Melanocytes against Visible Light-Induced Photodamage. Free Radical Biology and Medicine, 131, 399-407. https://doi.org/10.1016/j.freeradbiomed.2018.12.028

[60] Luyckx, J. and Baudouin, C. (2011) Trehalose: An Intriguing Disaccharide with Potential for Medical Application in Ophthalmology. Clinical Ophthalmology, 5, 577-581. https://doi.org/10.2147/OPTH.S18827

[61] Bragg, J.T., et al. (2017) Esterified Trehalose Analogues Protect Mammalian Cells from Heat Shock. ChemBioChem, 18, 1863-1870. https://doi.org/10.1002/cbic.201700302

[62] Rodrigues, D., et al. (2016) Mechanism of Aloe Vera Extract Protection against UVA: Shelter of Lysosomal Membrane Avoids Photodamage. Photochemical \& Photobiological Sciences, 15, 334-350. https://doi.org/10.1039/C5PP00409H 